\title{
RECURRENCE AND PRESERVATION OF MEASURE
}

\section{F. H. SIMONS}

Let $T$ be a measurable (not necessarily invertible) transformation in a (not necessarily $\sigma$-finite) measure space $(X, \Re, \mu)$. The following theorem extends results of Halmos-Ornstein [1] and Helmberg [2], while the proof is rather simple.

Theorem. Suppose $\mu\left(T^{-1} A\right) \leqq \mu(A)$ for all $A \in \Re$. If $E \in \Re$ satisfies $E \subset \cup_{n=1}^{\infty} T^{-n} E[\mu]$, then $\mu\left(T^{-1} E\right)=\mu(E)$.

Proof. Suppose $\mu\left(T^{-1} E\right)<\mu(E)$. Put $E_{1}=T^{-1} E \cap E$ and $A_{1}$ $=T^{-1} E \cap(X \backslash E)$, then $E_{1} \in \Re, A_{1} \in \Re$, and

$$
\mu(E)>\mu\left(E_{1}\right)+\mu\left(A_{1}\right) .
$$

The sets $E_{n} \in \Re, A_{n} \in \Re$ are defined inductively for $n \geqq 2$ by

$$
E_{n}=T^{-1} A_{n-1} \cap E, \quad A_{n}=T^{-1} A_{n-1} \cap(X \backslash E) .
$$

It follows that for every $n \geqq 2$

$$
\mu\left(A_{1}\right) \geqq \mu\left(E_{2}\right)+\cdots+\mu\left(E_{n}\right)+\mu\left(A_{n}\right)
$$

hence

$$
\mu\left(A_{1}\right) \geqq \sum_{n=2}^{\infty} \mu\left(E_{n}\right), \quad \mu(E)>\sum_{n=1}^{\infty} \mu\left(E_{n}\right) .
$$

Since $\cup_{n=1}^{\infty} E_{n}$ is the set of recurrent points of $E$, we must have $\mu\left(E \backslash \bigcup_{n=1}^{\infty} E_{n}\right)=0$. Contradiction.

In particular, if $T$ is also conservative, then $E \subset \cup_{n=1}^{\infty} T^{-n} E[\mu]$ for all $E \in \Re$, hence $T$ is measure preserving.

As a corollary we obtain by repeating the proof given in $[2, \S 4]$, that if $T$ is a conservative measure preserving transformation in $(X, \Re, \mu)$, for every $E \in \Re$ the induced transformation $T_{E}$ on $(E, \Re \cap E, \mu)$ is conservative and measure preserving.

\section{REFERENCES}

1. P. R. Halmos, Lectures on ergodic theory, Publ. Math. Soc. Japan, no. 3, The Mathematical Society of Japan and Chelsea, New York, 1960. MR 20 \#3958, MR 22 \#2677.

2. G. Helmberg, Über konservative Transformationen, Math. Ann. 165 (1966), 44-61. MR 33 \#5842.

Technological University, Eindhoven, Netherlands

Received by the editors May 10, 1968. 